\title{
Induction of cell differentiation and genomic stability in cancer, by an "multi epimutation-targeted therapy", or MTET
}

\author{
M Nezami $^{1 *}$, S Hager ${ }^{2}$ and A GouldSimon ${ }^{3}$ \\ ${ }^{1}$ President, Orange Coast Medical Center of Hope Inc., USA \\ ${ }^{2}$ Department of Oncology, cCARE, Fresno,CA 93720, USA \\ ${ }^{3}$ Department of Nuclear Medicine, Valley Metabolic Imaging LLC, Fresno, CA 93710, USA
}

\begin{abstract}
This article reviews and classifies epigenetic alterations driven by the induction of hypoxia, which are involved with genomic instability. We explore the biological relationship of key targets involved in genomic instability and heterogeneity in cancer. Subsequently, in order to reverse the key translational biologic events in tumorogenesis and metastasis, we propose a method which induces cell differentiation, by epigenetic modification. We correlate important genomic mutations, or methylations, with subsequent epimutational events that cause instability in cancer. We hypothesize that inhibition of these epigenetic aberrancies should be selective in order to overcome genetic instability. Further, we discuss our findings in preclinical and clinical settings related to our hypothesis and conclude that such an approach may overcome the current therapeutic challenges in refractory solid tumors with genomic instability.
\end{abstract}

\section{Background}

Recent research has opened scientists' eyes to look deeper into biology of cancer cell and describe epigenetic networks, which drive tumor cells. Recent work connects epigenetics to stem cell plasticity and carcinogenesis, beyond what the genomics of a cell could explain. Although exciting, this new perspective of medicine brings complexity into clinical approaches, as it challenges the traditional concepts of cancer biology by discovering the nature of evolutionary epigenetics, which still remains to be fully understood.

One of the main therapeutic challenges in identifying actionable targets to treat in cancer is the tumor genomic instability in concert with its stem cell plasticity and epigenomic instability. Literature regarding epimutations in cancer identifies common epigenomic signatures, such as $\mathrm{CpG}$ island methylator phenotype (CIMP) and its genomic equivalent, microsatellite instabilities (MSI). Many of the epigenetic mutations (epimutations) can be the cause of this pattern. For example, IDH1, (Tet2 mutations), are epigenetic mutations in the case of CpG island mutated phenotype (CIMP), and inactivating Set Domain Containing 2 (SETD2) mutations are reported in the case of MSI [1-11]. Also, some evidence has suggested that MLH1 mutations and epimutations can reciprocally influence each other and suggest that an altered structure of the MLH1 locus results in epigenetic alteration [5]. For example, extensive promoter methylation is associated with MLH1 inactivation, MSI, and BRAF mutation in colon cancer [3]. Somatic MLH1 hypermethylation has been detected in $91.9 \%$ of cases in some studies, with MSI BRAF-mutated CRC [4].

In general, epigenomic mutations occur with higher incidence than genomic errors. Although reversible through generations, their penetration is sometimes enough to cause cancer by itself, without further epigenetic dysregulation. For example, IDH1 mutations are sometimes enough to cause sarcomas [2]. The investigational drugs that inhibit the epigenetic drivers such as Tet2, IDH1, and DNMT have widely failed in solid tumors. The challenge in therapeutic approaches are not only the fact that each epigenetic target needs to be verified, and inhibited if mutated, but also that the downstream epigenetic targets would have to be selectively targeted to accomplish response. This cannot be achieved through the use of available demethylators such as decitabine or azacytidine, as they are non-selective in their targets.

\section{Hypothesis}

To identify the key epigenetic targets in inducing genomic instability we selected SETD2, a common mutated epigene in colorectal cancer, acting as an $\mathrm{H} 3 \mathrm{~K} 36$ methyltransferase. This is one of the key epigenetic targets that is inactivated by mutation. This occurs in variety of solid tumors and correlates with microsatellite instability. SETD2-dependent histone $\mathrm{H} 3 \mathrm{~K} 36$ trimethylation is required for homologous recombination repair and genome stability [6]. Histone modifications establish the chromatin states that coordinate the DNA damage response. It is also suggested that SETD2, is required for ATM activation upon DNA double-strand breaks (DSBs). In ccRCC, loss of SETD2 may afford an alternative mechanism for the inactivation of the p53-mediated checkpoint without the need for additional genetic mutations in TP53 [8]. The DNA double-strand break (DSB), arising from exposure to ionizing radiation or various chemotherapeutic

${ }^{\star}$ Correspondence to: $\mathrm{M}$ Nezami, President, Orange Coast Medical Center of Hope Inc., 496 Old Newport Blvd. \#7 Newport Beach, CA 92612, USA, E-mail: amnezami@yahoo.com

Key words: epimutation, epigenetic therapy

Received: September 05, 2018; Accepted: September 12, 2018; Published: September 14, 2018 
agents or from replication fork collapse, is among the most dangerous of chromosomal lesions. Mutations in SETD2 can actually cause a novel overgrowth condition [6,7,9]. Besides the reported H3K36trimethylation pathway, it is reported that SETD2 mutation also mediates mismatch repair gene (MMR) via AKT-induced PMS2 decrease and co-loss of MLH1 loss in ccRCC. Renal clear cell carcinoma (ccRCC) is characterized by frequent mutation in SETD2, which has recently been shown to regulate mismatch repair (MMR). In the presence of a genetic lesion, downregulation of SETD2 contributes to both initiation and progression during leukemia development by promoting the selfrenewal potential of leukemia stem cells [10].

This target is of key interest, as our investigation allowed us to correlate this as the most likely genomic mutational event in relation to carcinogenesis through this epigenetic pathway. We have identified that greater than $88 \%$ of mutations in this gene are accompanied by VHL inactivation, and PHD overexpression [1]. VHL inactivation appears to be an actionable target through inhibition of HIF-1, as we showed significant inhibition in our invitro studies using our epigenetic protocol (MTET).

VHL inhibition potentiates the HIF response element interaction and EMT transition through Proryl hydroxylases (PHD). It also increases stem cell plasticity through HIF2 alpha activation and Oct4 [1]. We also know that VHL inactivation can induce other epimutations such as EZH-2 which enhances the methylation of H3K29 and overexpression of DNMT3B and Methyl CpG island protein domain 1 (Mbd1). This correlates with, and interacts with, (if not causes) histone demethylation at H3k4, 36 and 79. Certainly the methylation of H3k9, 27 and 20 have the opposite effect, and so is the deacetylation of H4k16 and H3k9, commonly seen in cancer [1]. Inactivation of VHL also can cause changes in micro RNAs. Since VHL plays such a key regulator in inducing a panepigenomic and genomic effect, through HIF-1, we believe that a targeted therapy that inhibits the methylation of this target, would significantly reduce the presence of genomic instability, stem cell plasticity and heterogeneity in cancer.

\section{Methods}

We used in vitro application of our compounds consisting of one histone deacetylase inhibitor and one demethylator (Quercetin a polyphenol and Sodium Phenyl butyrate, a known HDACI) and we showed synergistic HIF inhibition in variety of cancer cell lines (Figure 1).

In vivo, we studied several cases of advanced solid tumor through their tumor liquid biopsy signature and we correlated their circulating tumor DNA (ctDNA) as a marker for heterogeneity and genomic instability with their response to therapy. Here we present these cases in detail and elaborate on the findings.

\section{Case Series}

\section{Case 1}

A 90-year old female with history of pancreatic cancer was referred to our clinic for evaluation and management. She was diagnosed in September 2015 and had not yet seen an oncologist due to insurance issues. She had not been staged by scans or had extensive blood workup. She had mild abdominal pain but was generally asymptomatic. She had been under the care of a gastroenterologist, and had a biliary stent placed. We referred her to an Oncologist, we drew her labs and ordered a PET scan. Her labs showed significantly elevated liver enzymes, bilirubin as well as ALK-P. Her PET scan showed an SUV of activity

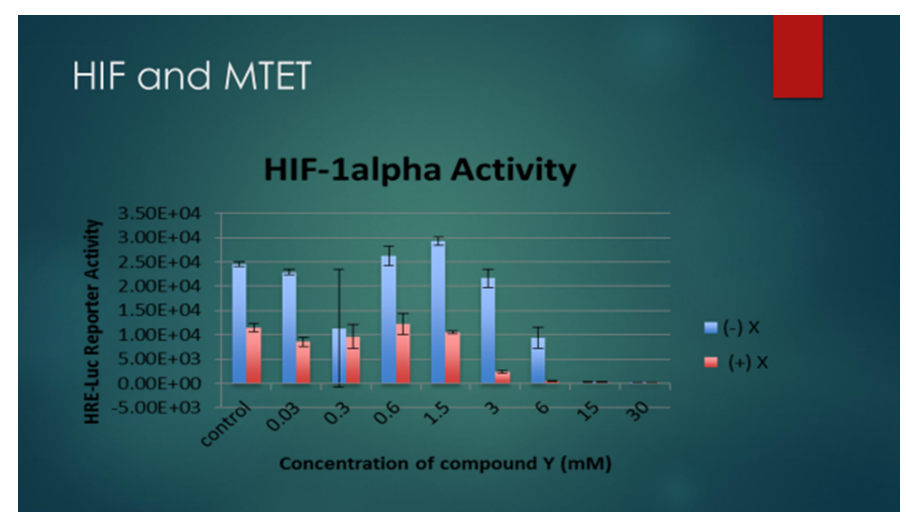

Figure 1. HIF inhibition by application of MTET

of 5.5 around the biliary stent. There were also retroperitoneal nodes with activity, as well as a solitary nodule in the liver that was likely metastatic. We also measured her cDNA using Guardant 360 labs, which was positive for KRAS as well as NF-1. Generally, when KRAS is mutated, we do not use erlotinib, but in her case, a combination of capecitabine and erlotinib was considered. She received IV epigenetic therapy daily for 10 treatments and tolerated it without side effects. She noted improved quality of life and energy. After 10 treatments, cDNA was repeated and showed that KRAS was no longer detected (Figure 2).

\section{Case 2}

A 37-year-old-female with history of breast cancer diagnosed in January 2014, Stage II A, ER/PR positive/Her 2 negative, presented to our clinic after trying and failing multiple alternative treatment modalities. She had previously refused all conventional therapies, including hormonal blockade. She had opted for alternative treatments in Mexico, and had exhausted Insulin Potentiated Chemotherapy (IPT), Vitamin C IV drip, and other alternative treatments, including autologous immune cell therapy and hyperthermia. She was referred to us by her PMD. She was status post mastectomy done in January 2014 and had recurrence of the tumor in September 2015 at the surgical implant replacement. She was being treated at the Cleveland clinic. Imaging done in November 2015 had confirmed presence of innumerable bony metastases as well as thoracic and neck lymph involvements, extensive chest and bone disease. Her right breast mass had an SUV of 14.8, and there were several axillary, mediastinal and hilar lymphadenopathies ranging in SUV of 7-9.6. Many sclerotic bony lesions in her glenoid, sternum and sacrum, with SUVs ranging between 7.8 to 9.2 were noted.

Upon her evaluation, we found significantly elevated tumor markers, as well as an elevated IGF-1. Her circulatory DNA by liquid biopsy was positive and showed with a mutated P53 as well as ERBB2. Her serum HER-2 was also elevated (Figure 3).

Her treatment plan included IV epigenetic therapies, which she received on daily basis. Her IGF-1 was repeated after two weeks of therapy and it reduced from 240 to $193 \mathrm{ng} / \mathrm{mL}$ (normal $69-227 \mathrm{ng}$ / $\mathrm{mL}$ ) measured on $11 / 9 / 15$ and $11 / 23 / 15$, respectively. She reported that her quality of life improved, and she had not changed her diet or supplements.

She was started trastuzumab based on tumor molecular profiling showing overexpression of HER-2 as well as elevated serum HER-2. She concurrently received IV epigenetic therapies, based on the molecular profiling of her tumor showing overexpression of TET2. 


\begin{tabular}{|l|l|}
\hline DOB: & Test Number 2 \\
\hline
\end{tabular}

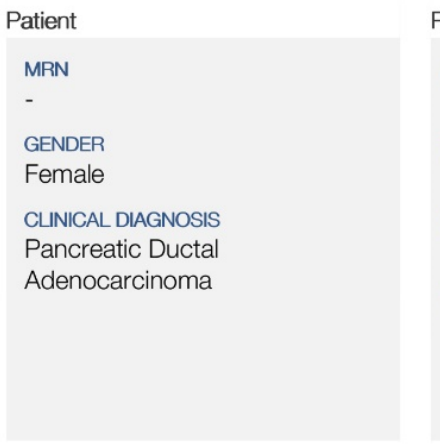

Reporting
REPORT DATE
DEC-28-2015
RECEIPT DATE
DEC-15-2015
COUECTION DATE
DEC-14-2015
SPECIMEN
Blood

Reporting

TATE

RECEIPT DATE

C-15-2015

Blood
Physician

NAME

Mohammed Amin Nezami

ACCOUNT

Pacific Medical Center of Hope

1680 E Herndon Ave Ste 102, Fresno, CA 93720

PHONE

FAX

$\begin{array}{ll}\text { (559) } 439-5393 & \text { (559) } 439-5828\end{array}$

ADDITIONAL RECIPIENT

Guardant360 Tumor Response Map

The Guardant360 Tumor Response Map illustrates the relative changes of observed cfDNA at different sample submission time points. The "Somatic Alteration Burden" value below refers to the maximum \% cfDNA detected at each time point. Amplifications are not plotted and only the first and last four test dates are plotted. Please see the physician portal for the Tumor Response Map with all test dates.

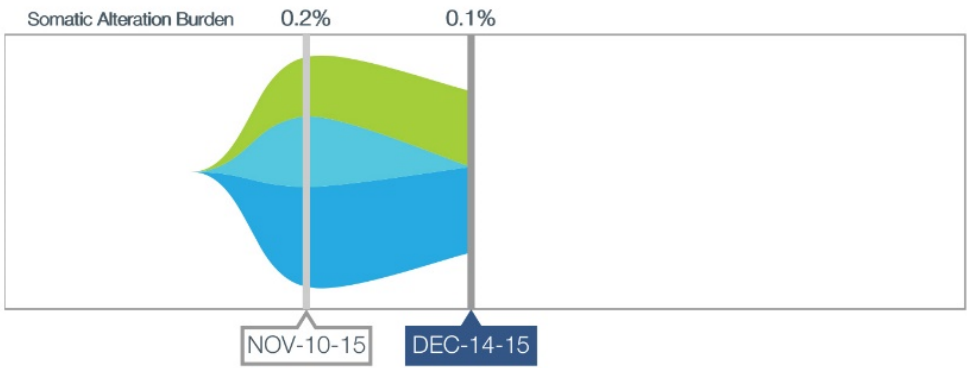

\section{Total Alteration(s) Detected}

0 with Associated Therapy

O Associated with Lack of Response

No Clinical Drug Trial Available

Summary of Alterations \& Associated Treatment Options

The percentage, or allele frequency, of altered cell-free DNA (\% cfDNA) circulating in blood is related to the unique tumor biology of this patient. Factors that may affect the amount/percentages of detected genomic alterations in circulating cell-free DNA in blood include tumor growth, turn-over, size, heterogeneity, vascularization, disease progression, or treatment.

\begin{tabular}{|c|c|c|c|c|c|c|c|}
\hline Alteration & & Mutation Trend & $\%$ cfDNA & cfDNA Amplification & $\begin{array}{l}\text { FDA Approved in } \\
\text { Indication }\end{array}$ & $\begin{array}{l}\text { Available for Use in } \\
\text { Other Indications }\end{array}$ & Clinical Drug Trials \\
\hline NF1 & L1187R & 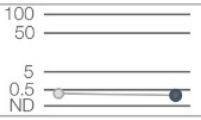 & 0.1 & \multicolumn{4}{|c|}{$\begin{array}{l}\text { The functional consequences and clinical significance of this gene variant are not } \\
\text { established. The relevance of therapies targeting this alteration is uncertain. Similar to } \\
\text { other alterations in circulating cfDNA, the monitoring of this variant may be reflective of } \\
\text { disease progression or treatment; clinical correlation is advised. }\end{array}$} \\
\hline FGFR2 & S110N & 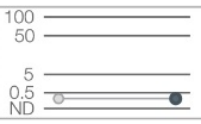 & 0.1 & \multicolumn{4}{|c|}{$\begin{array}{l}\text { The functional consequences and clinical significance of this gene variant are not } \\
\text { established. The relevance of therapies targeting this alteration is uncertain. Similar to } \\
\text { other alterations in circulating cfDNA, the monitoring of this variant may be reflective of } \\
\text { disease progression or treatment; clinical correlation is advised. }\end{array}$} \\
\hline KRAS & $G 12 D$ & $\begin{array}{l}100 \bar{\square} \\
50 \bar{\square} \\
0.5 \overline{\text { ND }}\end{array}$ & ND & & & & \\
\hline
\end{tabular}

For a more detailed Guardant360 Patient Report, log onto: https://portal.guardanthealth.com

To set up an account, contact Client Services: 855.698.8887

The chart above annotates the percentage or allele frequency, of altered circulating cell-free DNA (\% cfDNA) detected in this patient. The detected genomic alterations are listed in descending order by \% cfDNA by gene.

Genomic Alterations: Not Detected (ND). Genomic alterations may be present that are below the limit of detection of this test. Certain sample or variant characteristics may result in reduced analytic sensitivity, such as poor sample quality or improper collection. Genomic alterations in a tumor may be present, but are not detected in circulating cellfree DNA from this blood specimen with this test.

The "FDA Approved in Indication" and "Available for Use In Other Indications" columns describe drugs associated with specific genomic alterations. It is based on publicly available information as described in the "Detailed Therapy Results" and "Clinical Relevance of Detected Alterations" sections of the report.

GUARDANT HEALTH ${ }^{\oplus}$ Arthur Baca, MD PhD Laboratory Director | CLIA ID: 05D2070300 | 505 Penobscot Drive, Redwood City, CA 94063 Arthur Baca, MD PhD Laboratory Director | CLIA ID: 05D2070300 | 505 Penobscot Drive, Redwood City, CA 94063
T: 855-698-8887 | clientservices@guardanthealth.com | https://portal.guardanthealth.com | TST-PRT-001 V13.0|Pg 1 of 7

Figure 2. Case 1 


\section{Guardant360 Tumor Response Map}

The Guardant360 Tumor Response Map illustrates the mutant allele percentage (\% cfDNA) of observed somatic variants at each sample submission time point. Amplifications are not plotted, and only the first and last five test dates are plotted. Please see the Physician Portal (portal.guardanthealth.com) for the Tumor Response Map with all test dates.

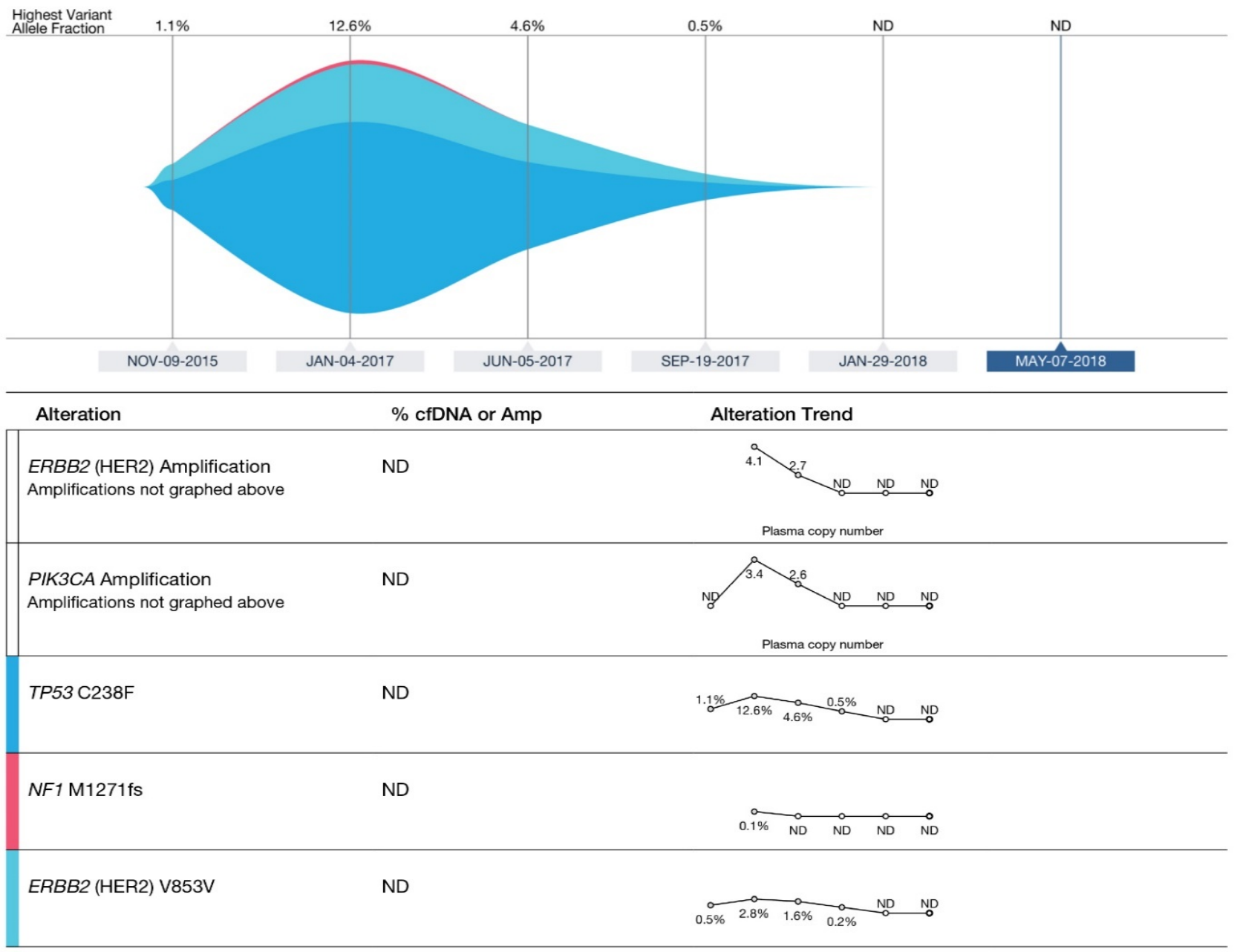

The table above annotates the variant allele fraction (\% cfDNA) detected in this sample, listed in descending order.

GUARDANT HEALTH ${ }^{*}$ A more detailed Guardant360 Patient Report is available through our online portal: TST-PRT-001 V17.0|Pg 2 of 4 portal.guardanthealth.com or to set up an account, contact Client Services: $\underline{\mathbf{8 5 5 . 6 9 8 . 8 8 8 7}}$

\section{Figure 3. Case 2}

Her CTC was initially positive for ERBB2, cMYC, and CK19, and was repeated by BioFocus on 12/17/15 and it showed complete eradication of CTC with CK20 and ERBB2. The cMYC virtually unchanged. Her LDH dropped from 303 to $147 \mathrm{UI} / \mathrm{L}$, after just three treatments, measured on $12 / 25$ and $2 / 28 / 15$ respectively.

Her circulatory DNA was measured through Guardant360, and it was positive for PI3K and TP53 alterations with elevated MAF, measured on $11 / 9 / 15,12 / 1 / 15$ and negative on $12 / 30 / 15$. She received trastuzumab along with IV epigenetic therapies, which could explain the disappearance of ERBB2 from her CTC and/or cDNA, however there are no expected positive effects from trastuzumab on P53, or PI3K, nor the cMYC present at her initial CTC. We contribute these results to the IV epigenetic therapies she received in the interim, as this therapy in fact targets cMYC, P53 and PI3K simultaneously.

On 1/20/2016, her CTC was repeated, and it showed complete resolution of CTC post therapy. She stopped the IV epigenetic therapies on in January 2016, as she moved back home to Cleveland, and stayed on trastuzumab every three weeks, along with monthly leuprolide and denosumab injections, and oral tamoxifen. Her restaging PET scan in August 2016 showed significant progression of her disease with increased sizes and metabolic activities in all her lesions in thoracic and bones. There was a new L1 lesion with SUV activity of 16.9. Her sternal lesion enlarged and had an SUV activity that increased to 16.9 from 5.6, as well as a sacral lesion that enlarged with SUV activity of 16.9 from 5.6. 
She immediately returned to our clinic. At this point the CTC was repeated, and it showed the presence of HER-2 positive cells again. She was started back on the IV therapies again on $8 / 25 / 16$, administered on daily basis. Her restaging scan was ordered on $9 / 15 / 16$ and showed partial response to therapy with significant reduction in all her lesions sizes and SUV metabolic activities. Her cervical nodes improved, right breast implant with SUV of 8.2 from 12.2, axillary nodes 4.2 down from 6.0, pulmonary hilar nodules 4.3 from 9.2, and bony lesions in sternum 10.2 from 16, L1 from 16.3 down to 11.9, right scapula from 13.9 down to 8.4 , and sacrum from 7.4 to 6.6 .

\section{Case 3}

A 50-year-old female with a suspicious left breast mass presented to us for evaluation and management in November 2015. She had not yet had a biopsy when she referred to us, and initially refused to do so. Her breast cancer tumor markers were elevated and had been drawn by her chiropractor after she found a mass in her left breast growing for over a year. A thermogram was performed which was suspicious. Upon her arrival to our clinic, we ordered biomarkers as well as a staging PET scan, which showed a significantly metabolically active tumor in her left breast as well as axilla, and SUV activity in her iliac bones as well as thoracic and lumbar spine. The most active lesion had an SUV activity of 9.9. Her tumor markers were extremely elevated and had positive cDNA on her liquid biopsy done by Guardant360, which was positive for PI3K as well as cKIT alterations, measured on 11/19/15. Her transforming growth factor (TGF), was also elevated, as expected from her cKIT overexpression. Her CTC came back positive for cMYC and for ERBB2 (HER-2). She eventually agreed to biopsy, which showed both ductal and lobular carcinoma that was ER positive and HER-2 negative.

She was started on combination of MTET and trastuzumab. Her TGF also dropped from 24,000 measured on 1/19/16 down to 1258 on $2 / 22 / 16$. Her cancer was restaged on $4 / 1 / 16$, through a whole-body PET, which showed a near complete resolution of thoracic lymph nodes by FDG activity, as well as complete resolution of FDG activity in T3 and L4 bony mets (the SUV dropped to 2.0 compared to 9.1). There were pulmonary nodules with remaining activity.

She was further started on everolimus in December 2016. Her cDNA was repeated, and it showed stable KIT, and resolution of PI3K to non-detectable (Figure 4). Further cDNA showed reduction of MAF to $0.5 \%$. She had another restaging PET scan on $1 / 3 / 17$, which showed improvement in both size and activity of all metastatic lesions in the lungs, and bony pelvis. No new lesion was seen. The left upper lobe lung lesion decreased in size from 1.4 to $1.2 \mathrm{~cm}$, and SUV from 12.6 to 4.9 , left upper lobe lesion $1 \mathrm{~cm}$ from $1.6 \mathrm{~cm}$, activity down from 11.7 to 1.1 , right upper lobe lung lesion $0.9 \mathrm{~cm}$ from $1.3 \mathrm{~cm}$, activity down from 10 to 3.7, axillary nodes SUV of 0.9. Her left iliac wing lesion decreased SUV activity from 8.3 to 4.7 . The left ilium decreased from 9.9 to 4.5 . The inferior pubic ramus decreased from 5.3 to 3 . The L4 lesion stable. Further she stopped the epigenetic therapies and continued with her conventional treatments with afinitor, her c DNA showed significant increase on May 22,2017 (Figure 4 and 5).

\section{Case 4}

An 80-year-old male with history of multiorgan failure, and locally advanced left posterior bladder cancer was referred to us for evaluation and management in June 2015. He had been suffering from partial obstruction of the rectum, secondary to inflammation versus malignancy in rectal region, confirmed by the pelvic CT. He also had renal failure that is also possibly secondary to bladder mass causing obstruction of urine flow, with hydronephrosis in both sides, right more than left, seen in his imaging. He also reported bad odor and mouth dryness, which I contribute to the uremia he has with majorly impaired kidney function. We ordered laboratory studies for him, including Guardant 360 for cDNA. His labs showed elevated CEA and CA19-9. His cDNA revealed many alterations including BRCA2, PI3K and P53. He was started on IV epigenetic therapies and antiinflammatory therapies aiming at reducing his pain and improving his creatinine. After 10 IV treatments, his cDNA was repeated, and showed reduced heterogeneity by disappearance of several alterations, as well as decreased MAF (Figure 6).

\section{Case 5}

A 54-year-old female with history of EGFR mutated lung adenocarcinoma was referred to us in February 2018. She had been on afatinib, which was started in 2016, and resulted in decreased CEA levels for several months, but now has progression of disease. Her oncologist ordered lab tests by Travogene, which were negative for any mutation in T790M. She is complaining of back pain at this time which is perhaps due to increased tumor growth at thoracic spine (T4), she also has noticed an enlarged Left axillary node for about 2 months. Her brain MRI was done in December 2017 and was normal. Our plan of care included IV epigenetic therapies, which we hoped would increase the response to the afatinib. After 10 treatments, her cDNA ws repeated, which showed improvement, with many alterations no longer detected, and her EGFR down to 5.5 MAF from 30.4 (Figure 7).

\section{Case 6}

A 57-year-old female with a history of invasive ductal breast carcinoma, presented for evaluation and management. She was first diagnosed in October 2000. She was treated with alternative treatments in Mexico and remained in remission until 2008, when she had right breast pain. She went back to Mexico and had right mastectomy for recurrent breast cancer. Her pathology showed ER/PR/HER2 triple positive with a Ki67 of $60 \%$. After that, she received trastuzumab and hormonal blockade with anastrozole. Her PET scan done at that time confirmed metastatic disease with bone involvement. She then was treated at St. Jude's where she continued hormonal blockade, trastuzumab, and zoledronic acid. She progressed slowly and was under the care of her local oncologist. She also tried and failed denosumab. She was started on tamoxifen and offered radiation. She had been switched to letrozole near the time of presentation, but review of her PET scan on 6/8/16 again showed progression of disease with complete destruction of the sternum. There were multiple lung metastases, as well as extensive disease in the peritoneum. There were no liver lesions noted. She has never had a paracentesis, although she appeared to have ascites. She referred to us seeking alternative and integrative treatments. Upon her arrival she was evaluated, and her labs drawn, which showed presence of CTCs in the blood, along with expression of ERBB2 and cMYC. These CTCs were ER negative.

Her molecular profiling was performed which showed presence of several mutations in her tissue biopsied from her humerus. First, they were HER-2 negative, second, they were ER positive. Third they were MSH-6 positive, making them responsive to the IV epigenetic therapies which started immediately on daily basis.

Her labs reported decreased tumor markers, CA 27.29 at 685 from 765, CA 15.3 at 533 from 574, serum HER-2 at 75 from 98, measured on $6 / 28 / 16$ after two weeks of therapy. Her cDNA showed a marked reduction in MAF, from 7.4 to 5.0 at the level of PI3K. It was repeated 

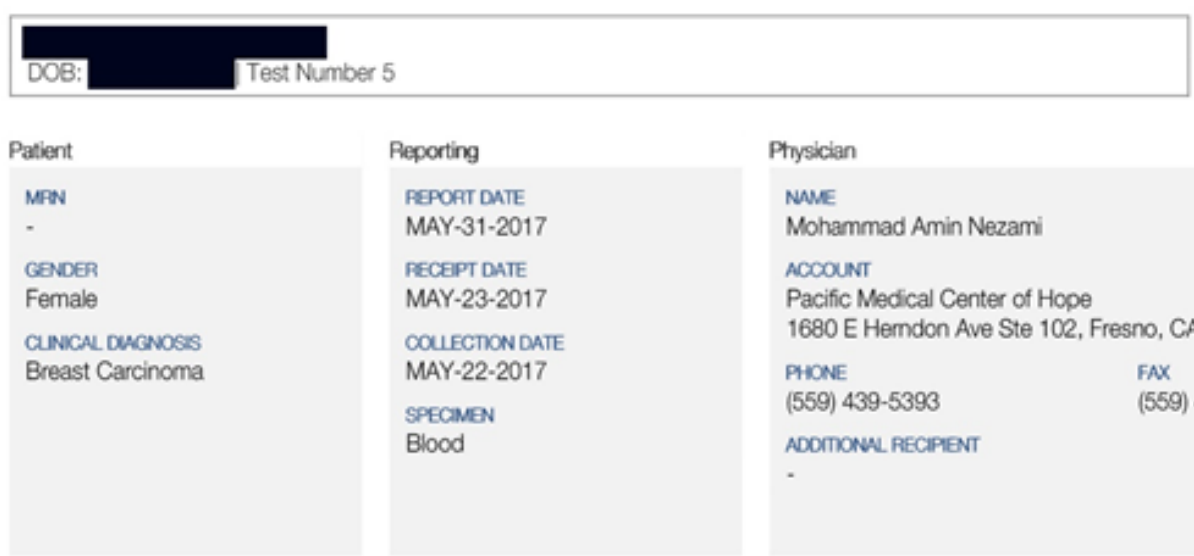

Reporting
PEPOAT DATE
MAY-31-2017
PECEPT DATE
MAY-23-2017
COUECTON DATE
MAY-22-2017
SPECMMEN
Blood

Blood

\author{
Prysician \\ Mohammad Amin Nezami \\ ACCOUNT \\ Pacific Medical Center of Hope \\ 1680 E Herndon Ave Ste 102, Fresno, CA 93720 \\ PHONE $\quad$ FAX \\ $\begin{array}{ll}\text { (559) } 439-5393 & \text { (559) } 439-5828\end{array}$ \\ ADOMONL RECIPIT
}

Guardant360 Tumor Response Map

The Guardant360 Tumor Response Map ilustrates the mutant allele percentage (\% CDNA) of cbserved scmatic variants at each sample submission time point. The "Somatic Aiteration Burden" value below refers to the maximum \% cfoNA detected at each time point. Amplifications are not pliotted, and only the first and last four test dates are plotted. Please see the Physician Portal (nttps:/portal.guardanthealth.com) for the Tumor Response Map with al test dates.

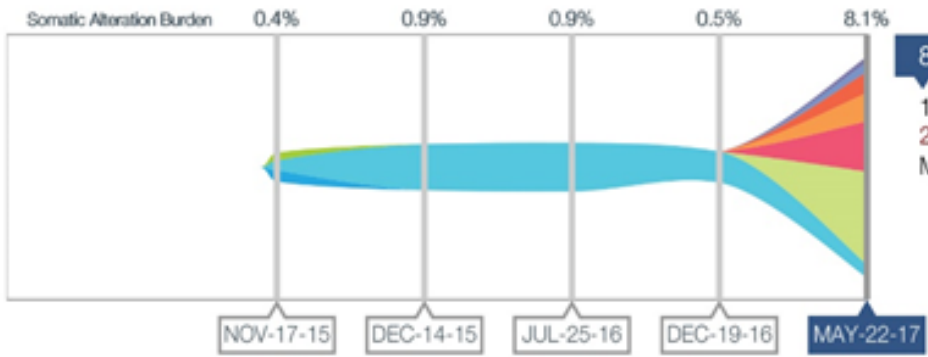

Summary of Somatic Alterations \& Associated Treatment Options

The percentage of altered cel-free DNA (\% cIDNA) croulating in bicod is related to the unique tumor biology of each patient. Factors that may affect the \% cfDNA of detected somatic alterations include tumor growh, tum-over, size, heterogeneity, vascularization, disease progression, and treatment.

\begin{tabular}{|c|c|c|c|c|c|c|}
\hline Aleration & & Mutasion Trend & $\begin{array}{l}\text { \% cfonu or } \\
\text { Ampification }\end{array}$ & $\begin{array}{l}\text { FDA Approwad in } \\
\text { indication } \\
\text { see pape } 4\end{array}$ & $\begin{array}{l}\text { Avelable for Use in } \\
\text { Other indications } \\
\text { see pape } 4\end{array}$ & $\begin{array}{l}\text { Chical Dng Triats } \\
\text { see pages }\end{array}$ \\
\hline \multicolumn{7}{|c|}{ Relevant for Therapy Selection } \\
\hline TP53 & $\begin{array}{l}\text { Sploo Site } \\
\text { SMV }\end{array}$ & $\begin{array}{r}100 \\
50= \\
05 \\
05\end{array}$ & 8.1 & None & None & Trats Avaliablo \\
\hline \multirow[b]{2}{*}{ ESAI } & Y537S & 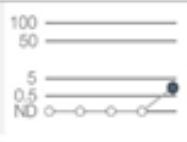 & 1.8 & $\begin{array}{l}\text { Lack of } \\
\text { Response: } \\
\text { Anastrozole, } \\
\text { Exemestane, } \\
\text { Letrozole }\end{array}$ & None & Trials Avalable \\
\hline & Y537N & $\begin{array}{l}100 \\
50 \\
0.5 \\
050000\end{array}$ & 1.2 & $\begin{array}{l}\text { Lack of } \\
\text { Pesponse: } \\
\text { Anastrozole, } \\
\text { Exemestane, } \\
\text { Letrozole }\end{array}$ & None & Trads Avalable \\
\hline
\end{tabular}

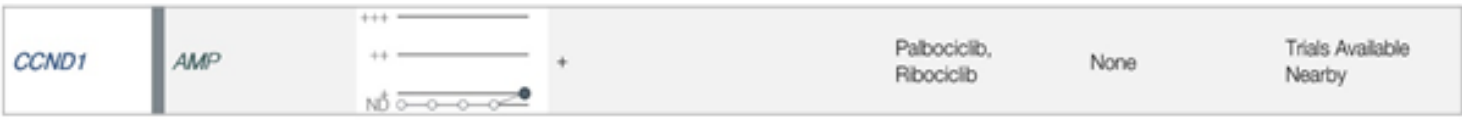

Figure 4. Case 3 


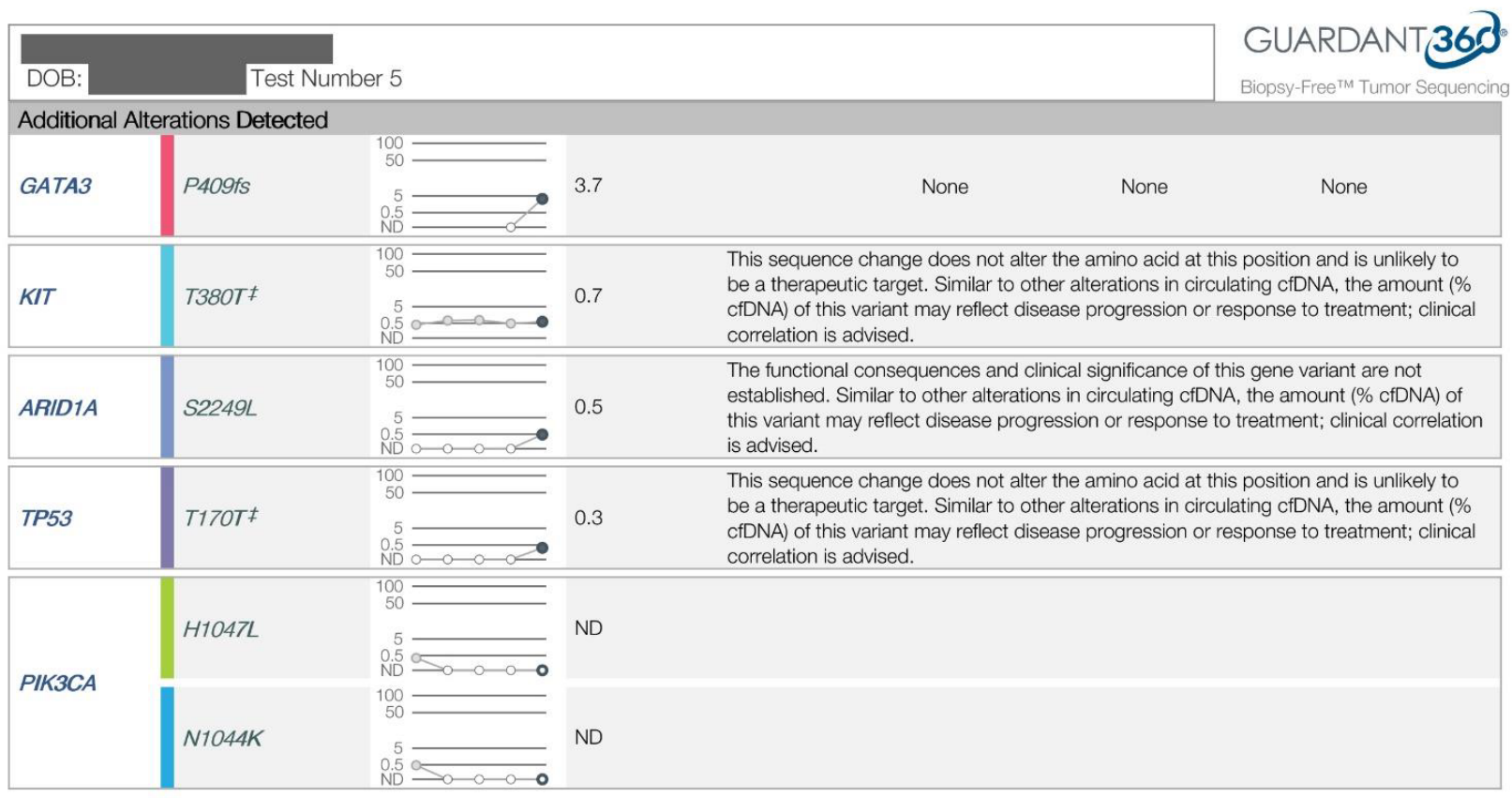

The chart above annotates the allele frequency of altered circulating cell-free DNA (\% cfDNA) detected in this patient. Alterations are listed in descending order of $\%$ cfDNA by gene.

All therapeutic annotations are based on publicly available information as described in the "Detailed Therapy Results" and "Clinical Relevance of Detected Alterations" sections of the report.

Genomic Alterations: Not Detected (ND). Genomic alterations may be present that are below the limit of detection of this test. Certain sample or variant characteristics may result in reduced analytic sensitivity, such as poor sample quality or improper collection. Genomic alterations in a tumor may be present, but are not detected in circulating cellfree DNA from this blood specimen with this test.

¥ Synonymous mutations and Variants of Uncertain Significance (VUSs): The functional consequences and clinical significance of this gene variant are not established. Similar to other alterations in circulating cfDNA, the amount (\% cfDNA) of this variant may reflect disease progression or response to treatment; clinical correlation is advised.

\section{Definitions}

Amplification: Gene amplification results in increased copies of the gene present in the cfDNA. As the absolute number of copies in circulation is dependent on both tumor fraction and the magnitude of the tumor amplification, amplifications are reported on a semi-quantitative scale: Positive (+): amplification magnitude is in the lower 50 th percentile of samples with amplifications; Strongly Positive (++): amplification magnitude is in the 50th to 90th percentile; Very Strongly Positive (+++): amplification magnitude is in the top 10th percentile. Guardant360 detects amplifications in the genes listed in Table 1.

Insertion (Ins): The following alteration was detected in this patient: GATA3 P409fs. Guardant360 detects short insertions in exons of certain genes (see Table 1).

Splice Site: Splice site variants disrupt the donor and/or acceptor splice site(s), leading to abnormal mRNA splicing and altered protein levels and/or function.

\section{Comments}

None.

Interpretation

Somatic alterations were detected in the circulating cell-free DNA isolated from this patient's blood specimen. These genomic alterations are cancer-associated somatic variants, some of which have been associated with either increased or reduced clinical response to specific treatments.

Amplification was detected in the circulating cell-free DNA isolated from this patient's blood specimen for the annotated gene(s). Unlike tissue-based gene amplification tests (e.g. IHC or FISH), Guardant360 assesses the total representation of a given gene in all circulating cell-free DNA present in the patient's blood sample including material derived from the tumor and healthy tissue alike. As such, the absolute level of amplification present in the blood depends both on the tumor-derived cfDNA content and on the degree of amplification within that fraction and cannot be inferred from bulk cfDNA interrogation. For example, a positive Guardant360 test could represent a small population of cells with extremely high levels of the detected gene amplification. Alternatively, it could represent a large population of cells with low to medium levels of the detected gene amplifications. The exact correlation between amplification detected by Guardant360 compared to IHC or FISH and how each test differentially guides patient management is an area of active investigation.

GUARDANTHEALTH For a more detailed Guardant360 Patient Report, log onto: https://portal.guardanthealth.com or to set up an account, contact Client Services: 855.698.8887

Figure 5. Test results of figure 4 


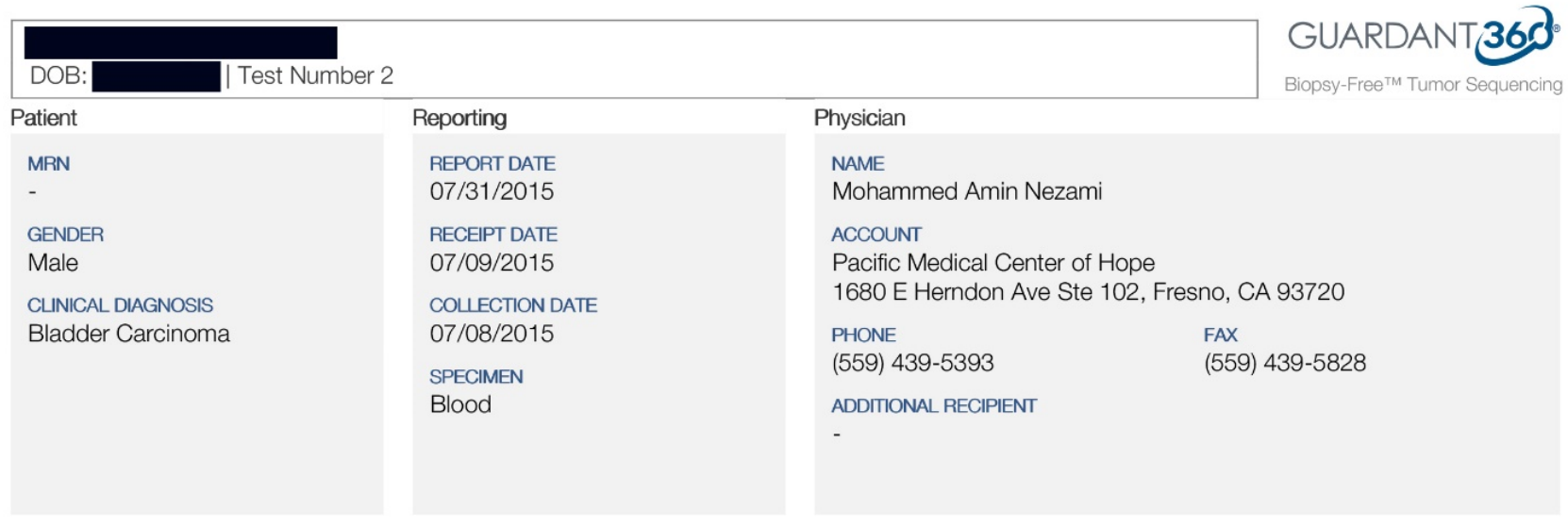

\section{Guardant360 Tumor Response Map}

The Guardant360 Tumor Response Map illustrates the relative changes of observed cfDNA at different sample submission time points. The "Somatic Alteration Burden" value below refers to the maximum $\%$ cfDNA detected at each time point. Amplifications are not plotted.

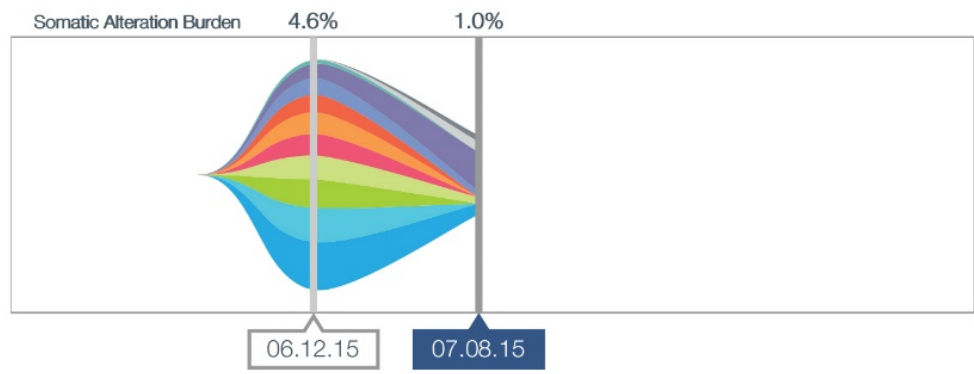

\section{Total Alteration(s) Detected}

1 with Associated Therapy

0 Associated with Lack of Response

Multiple Clinical Trials Available

Summary of Alterations \& Associated Treatment Options

The percentage, or allele frequency, of altered cell-free DNA (\% cfDNA) circulating in blood is related to the unique tumor biology of this patient. Factors that may affect the amount/percentages of detected genomic alterations in circulating cell-free DNA in blood include tumor growth, turn-over, size, heterogeneity, vascularization, disease progression, or treatment.

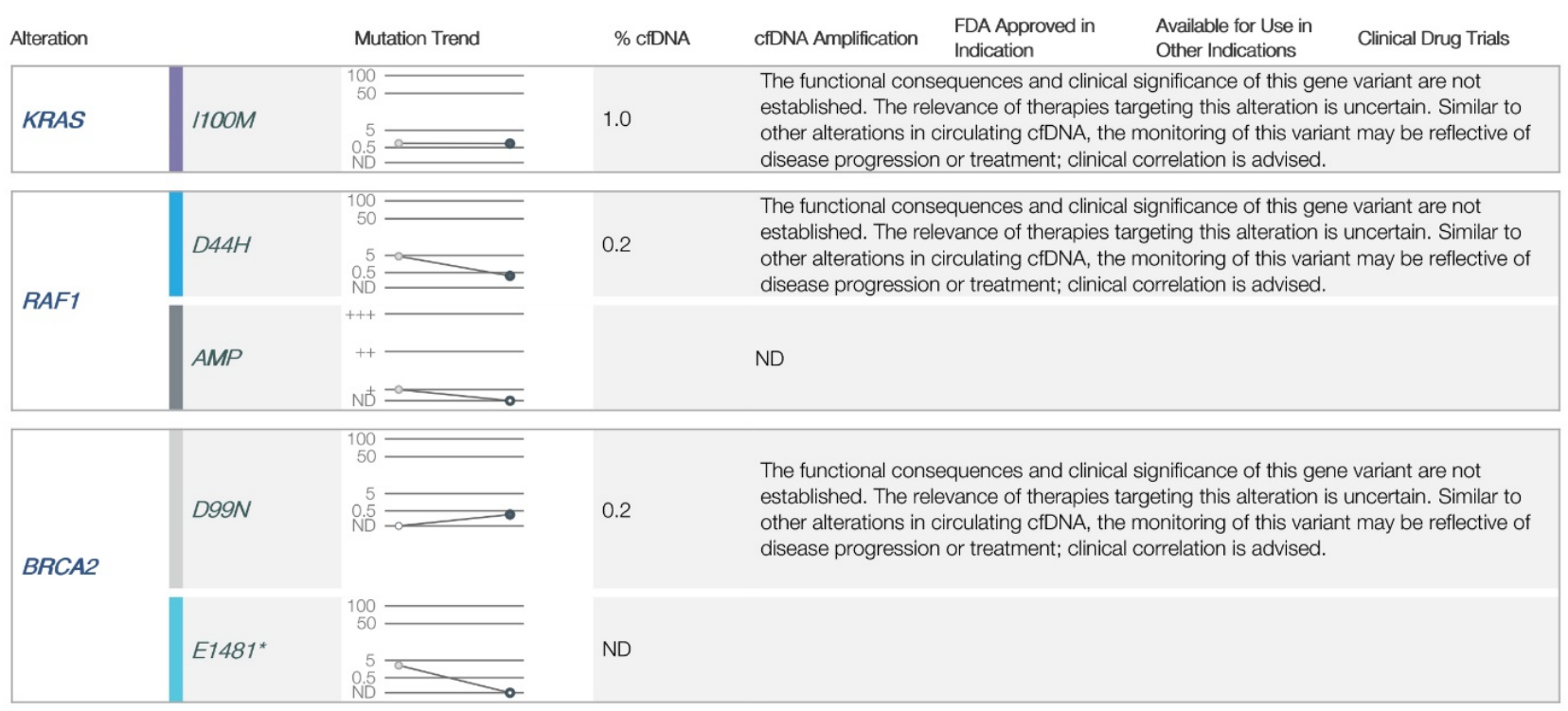
\begin{tabular}{l|l}
\hline GUARDANT HEALTH & $\begin{array}{l}\text { Arthur Baca, MD PhD Laboratory Director | CLIA ID: 05D2070300 | } 2686 \text { Middlefield Rd, Suite C, D, E, Redwood City, CA 94063 } \\
\text { T: 855-698-8887 | clientservices@guardanthealth.com | https://portal.guardanthealth.com | Report Version 4.0 | TST-PRT-001 V11.0 |Pg 1 of 15 }\end{array}$
\end{tabular}

Figure 6. Case 4 


\begin{tabular}{|l|l|}
\hline DOB: & Test Number 2 \\
\hline
\end{tabular}

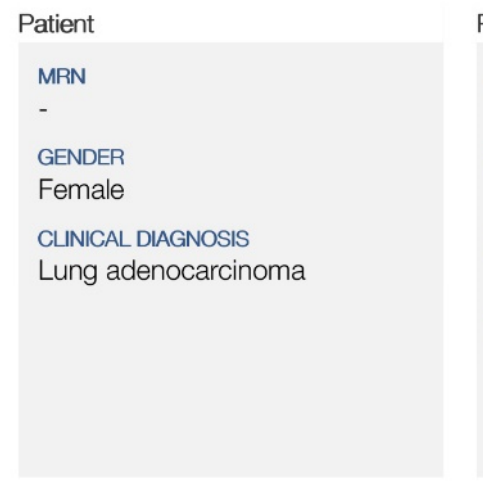

Reporting
REPORT DATE
MAR-17-2018
RECEIPT DATE
MAR-10-2018
COUECTION DATE
MAR-09-2018
SPECIMEN
Blood

Blood
Physician

NAME

Mohammad Amin Nezami

ACCOUNT

Pacific Medical Center of Hope

1680 E Herndon Ave Ste 102, Fresno, CA 93720

PHONE

(559) 439-5393

FAX

(559) 439-5828

\section{Guardant360 Tumor Response Map}

The Guardant360 Tumor Response Map illustrates the mutant allele percentage (\% cfDNA) of observed somatic variants at each sample submission time point. The "Somatic Alteration Burden" value below refers to the maximum \% cfDNA detected at each time point. Amplifications are not plotted, and only the first and last four test dates are plotted. Please see the Physician Portal (https://portal.guardanthealth.com) for the Tumor Response Map with all test dates.

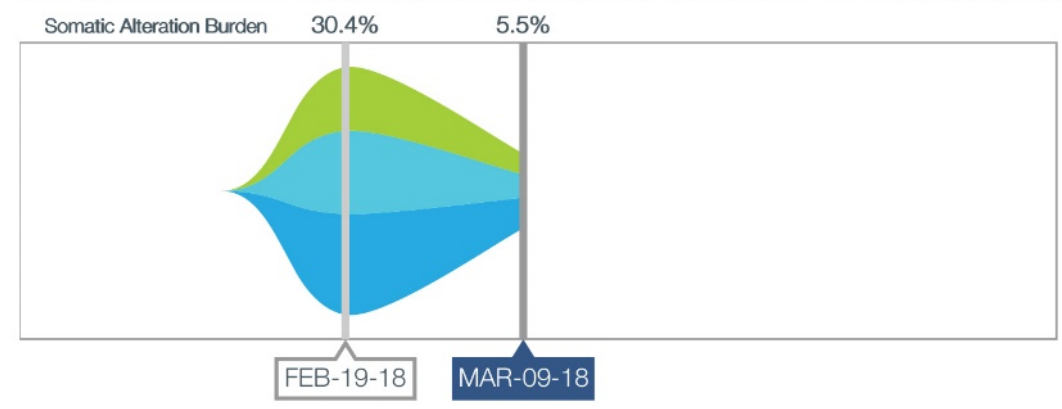

\section{Total Somatic Alteration(s) Detected}

4 with Associated Therapy

1 Associated with Lack of Response

Multiple Clinical Trials Available

\section{Summary of Somatic Alterations \& Associated Treatment Options}

The percentage of altered cell-free DNA (\% cfDNA) circulating in blood is related to the unique tumor biology of each patient. Factors that may affect the \% cfDNA of detected somatic alterations include tumor growth, turn-over, size, heterogeneity, vascularization, disease progression, and treatment.

\begin{tabular}{|c|c|c|c|c|c|}
\hline Alteration & Mutation Trend & $\begin{array}{l}\% \text { cfDNA or } \\
\text { Amplification }\end{array}$ & $\begin{array}{l}\text { FDA Approved in } \\
\text { Indication } \\
\text { see page } 3\end{array}$ & $\begin{array}{l}\text { Available for Use in } \\
\text { Other Indications } \\
\text { see page } 3\end{array}$ & $\begin{array}{l}\text { Clinical Drug Trials } \\
\text { see page } 9\end{array}$ \\
\hline
\end{tabular}

Figure 7. Case 5

after continued treatment and showed more reduction from 5 to 1.9 , measured on $8 / 2 / 16$.

She received trastuzumab after two weeks of MTET. Her initial response to MTET was reduction of her serum HER-2 level.

She did not respond to either lapatinib or trastuzumab, as her serum HER-2 increased again. Since this seemed to be a case of inherent resistance to trastuzumab, she was started on epigenetic therapies again, which resulted in decreased serum HER-2. It was decided to use afatinib (a pan-EGFR blocker) in conjunction with trastuzumab, in her case. Her tumor markers dropped, and she continued to respond to therapy with improved markers and her c DNA continued to improve with reduced PI3k and CCND1, measured on August 2016 (Figure 8).

\section{Conclusion}

We propose further trials based on our promising findings in our samples using MTET protocol to target VHL/ HIF pathway. We also recommend further generation of hypothesis based on the clinical model of response which includes application of companion diagnostic tools such as circulating DNA. 


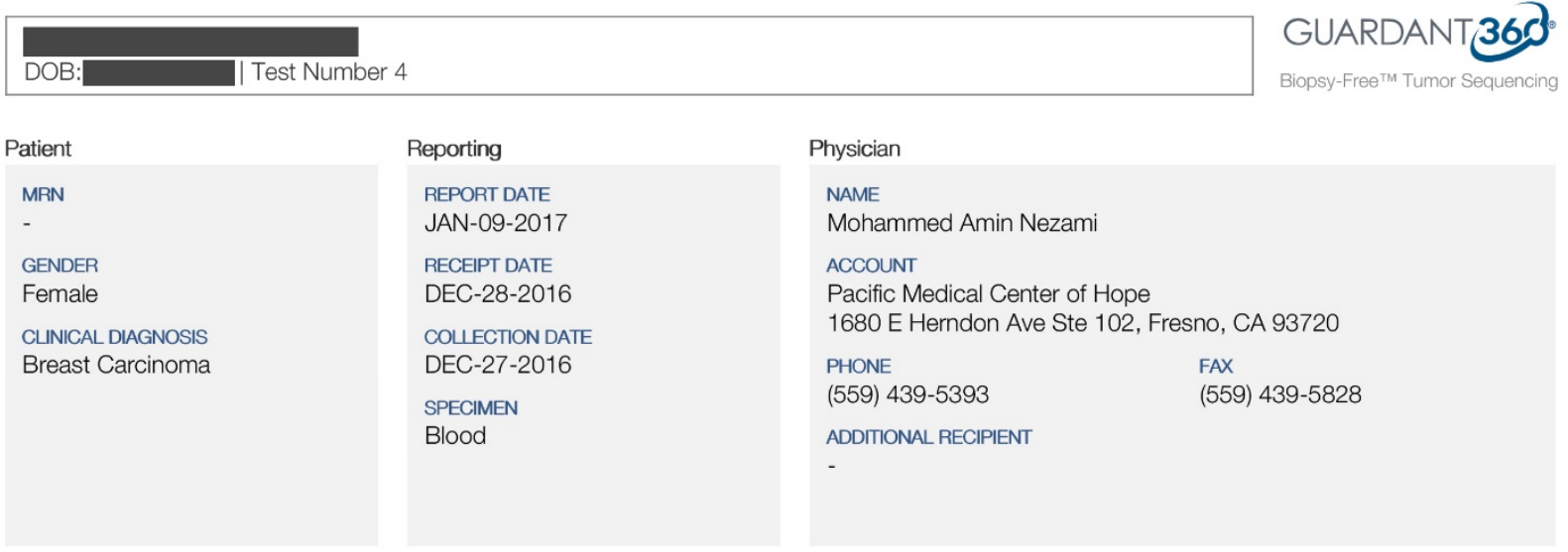

Guardant360 Tumor Response Map

The Guardant 360 Tumor Response Map illustrates the mutant allele percentage (\% cfDNA) of observed somatic variants at each sample submission time point. The "Somatic Alteration Burden" value below refers to the maximum \% cfDNA detected at each time point. Amplifications are not plotted, and only the first and last four test dates are plotted. Please see the Physician Portal (https://portal.guardanthealth.com) for the Tumor Response Map with all test dates.

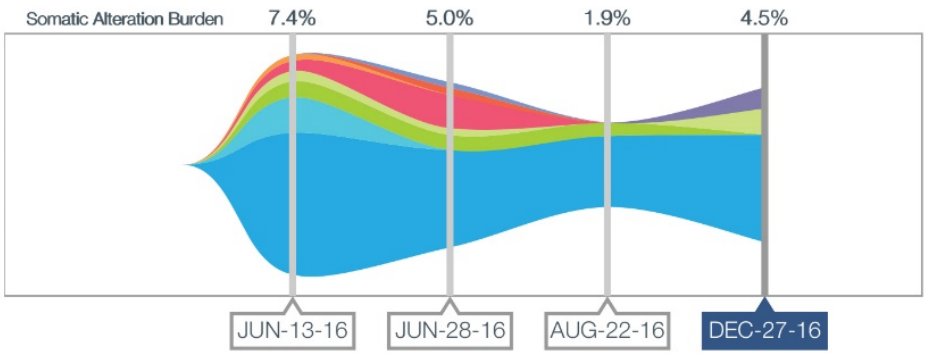

4 Total Somatic Alteration(s) Detected

3 with Associated Therapy

1 Associated with Lack of Response

Multiple Clinical Trials Available

Summary of Somatic Alterations \& Associated Treatment Options

The percentage of altered cell-free DNA (\% cfDNA) circulating in blood is related to the unique tumor biology of each patient. Factors that may affect the $\%$ cfDNA of detected somatic alterations include tumor growth, turn-over, size, heterogeneity, vascularization, disease progression, and treatment.

\begin{tabular}{|c|c|c|c|c|c|c|}
\hline \multicolumn{2}{|l|}{ Alteration } & Mutation Trend & $\begin{array}{l}\% \text { cfDNA or } \\
\text { Amplification }\end{array}$ & $\begin{array}{l}\text { FDA Approved in } \\
\text { Indication } \\
\text { see page } 4\end{array}$ & $\begin{array}{l}\text { Available for Use in } \\
\text { Other Indications } \\
\text { see page } 4\end{array}$ & $\begin{array}{l}\text { Clinical Drug Trials } \\
\text { see page } 9\end{array}$ \\
\hline \multicolumn{7}{|c|}{ Relevant for Therapy Selection } \\
\hline \multirow{2}{*}{ PIK3CA } & E542K & $\begin{array}{l}100 \\
50= \\
5.5 \square\end{array}$ & 4.5 & None & $\begin{array}{l}\text { Everolimus, } \\
\text { Temsirolimus }\end{array}$ & $\begin{array}{l}\text { Trials Available } \\
\text { Nearby }\end{array}$ \\
\hline & E453Q & $\begin{array}{r}100 \\
50= \\
5 \\
0.5 \\
\mathrm{ND}\end{array}$ & 0.6 & None & $\begin{array}{l}\text { Everolimus, } \\
\text { Temsirolimus }\end{array}$ & Trials Available \\
\hline ESR1 & Y537N & $\begin{array}{l}100 \\
50 \\
\\
0.5 \\
\text { ND }=\square 00\end{array}$ & 0.8 & $\begin{array}{l}\text { Lack of } \\
\text { Response: } \\
\text { Anastrozole, } \\
\text { Exemestane, } \\
\text { Letrozole }\end{array}$ & None & $\begin{array}{l}\text { Trials Available } \\
\text { Nearby }\end{array}$ \\
\hline
\end{tabular}

\begin{tabular}{|l|llllll}
\hline CCND1 & AMP & Palbociclib None & Trials Available \\
Nearby
\end{tabular}

GUARDANTHEALTH ${ }^{\circ}$ For a more detailed Guardant360 Patient Report, log onto: https://portal.guardanthealth.com or to set up an account, contact Client Services: 855.698.8887 Arthur Baca, MD PhD Laboratory Director | CLA ID: 05D2070300 | 505 Penobscot Drive, Redwood City, CA 94063

Figure 8. Case 6 


\section{References}

1. Thirlwell C, Schulz L, Dibra H, Beck S (2011) Suffocating cancer: Hypoxia-associated epimutations as targets for cancer therapy. Clinical Epigenetics 3: 9. [Crossref]

2. Roy DM, Walsh LA, Chan TA (2014) Driver mutations of cancer epigenomes. Protein \& Cell 5: 265-296. [Crossref]

3. Vilkin A, Niv Y, Nagasaka T, Morgenstern S, Levi Z, et al. (2008) T2093 Microsatellite Instability (MSI), Hmlh1 Promoter Hypermethylation and BRAF Mutation Analysis in Sporadic Colorectal Cancers (Crcs) of Different Ethnic Groups in Israel. Gastroenterology 134. [Crossref]

4. Moreira L, Muñoz J, Cuatrecasas M, Quintanilla I, Leoz ML (2014) Prevalence of somatic mutl homolog 1 promoter hypermethylation in Lynch syndrome colorectal cancer. Cancer 121: 1395-1404. [Crossref]

5. Cini G, Carnevali I, Quaia M, Chiaravalli AM, Sala P (2015) Concomitant mutation and epimutation of the MLH1 gene in a Lynch syndrome family. Carcinogenesis 36 : 452-458. [Crossref]
6. Pfister S, Ahrabi S, Zalmas L, Sarkar S, Aymard F (2014) SETD2-Dependent Histone H3K36 Trimethylation Is Required for Homologous Recombination Repair and Genome Stability. Cell Reports 7: 2006-2018. [Crossref]

7. Clark A, Low K (1988) Pathways and Systems of Homologous Recombination in Escherichia coli. The Recombination of Genetic Material 155-215.

8. Carvalho S, Vítor A, Sridhara S, Martins F, Raposo A (2018) SETD2 is required for DNA double-strand break repair and activation of the p53-mediated checkpoint. [Crossref]

9. Luscan A (2018) Mutations in SETD2 cause a novel overgrowth condition. [Crossref]

10. Zhu X, He F, Zeng H, Ling S, Chen A, et al. (2018) Identification of functional cooperative mutations of SETD2 in human acute leukemia. [Crossref]

11. Feng C, Wen H (2015) MP39-03 Loss of MLH1 confers resistance to pi3k $\beta$ inhibitors in renal clear cell carcinoma with SETD2 mutation. The Journal of Urology 193.

Copyright: $\odot 2018$ Nezami M. This is an open-access article distributed under the terms of the Creative Commons Attribution License, which permits unrestricted use, distribution, and reproduction in any medium, provided the original author and source are credited. 